\title{
CBT and CFT for Chronic Pain
}

\author{
Graham Hadley ${ }^{1}$ - Matthew B Novitch ${ }^{2}$
}

Accepted: 12 February 2021 / Published online: 1 April 2021

(C) The Author(s), under exclusive licence to Springer Science+Business Media, LLC, part of Springer Nature 2021

\begin{abstract}
Purpose of Review Chronic pain is a widespread public and physical health crisis, as it is one of the most common reasons adults seek medical care and accounts for the largest medical reason for disability in the USA (Glombiewski et al., J Consult Clin Psychol. 86(6):533545, 2018; Schemer et al., Eur J Pain. 23(3):526-538, 2019). Chronic pain is associated with decreased functional status, opioid dependence and substance abuse disorders, mental health crises, and overall lower perceived quality of life (Korff et al., J Pain. 17(10):1068-1080, 2016). For example, the leading cause of chronic pain and the leading cause of long-term disability is low back pain (LBP) (Bjorck-van Dijken et al. J Rehabil Med. 40:864-9, 2008). Evidence suggests that persistent low back pain (pLBP) is a multidimensional biopsychosocial problem with various contributing factors (Cherkin et al., JAMA. 315(12):1240-1249, 2016). Emotional distress, pain-related fear, and protective movement behaviors are all unhelpful lifestyle factors that previously were more likely to go unaddressed when assessing and treating patient discomfort (Pincus et al., Spine. 38:2118-23, 2013). Those that are not properly assisted with these psychosocial issues are often unlikely to benefit from treatment in the primary care setting and thus are referred to multidisciplinary pain rehabilitation physicians. This itself increases healthcare costs, and treatments can be invasive and have risks of their own. Therefore, less expensive and more accessible management strategies targeting these psychosocial issues should be started to facilitate improvement early. As a biopsychosocial disorder, chronic pain is influenced by a range of factors including lifestyle, mental health status, familial culture, and socioeconomic status. Physicians have moved toward multi-modal pain approaches in order to combat this public health dilemma, ranging from medications with several different mechanisms of action, lifestyle changes, procedural pain control, and psychological interventions (Fashler et al., Pain Res Manag. 2016:5960987, 2016). Part of the rehabilitation process now more and more commonly includes cognitive behavioral and cognitive functional therapy. Cognitive functional therapy (CFT) and cognitive behavioral therapy (CBT) are both multidimensional psychological approaches to combat the mental portion of difficult pain control. While these therapies are quite different in their approach, they lend to the idea that chronic pain can and should be targeted using coping mechanisms, helping patients understand the pathophysiological process of pain, and altering behavior.

Recent Findings CFT differs from CBT functionally, as instead of improving managing/coping mechanisms of pain control from a solely mental approach, CFT directly points out maladaptive behaviors and actively challenges the patient to change them in a cognitively integrated, progressive overloading functional manner (Bjorck-van Dijken et al. J Rehabil Med. 40:864-9, 2008). This allows CFT to be targeted to each individual patient, with the goal of personalized reconceptualization of the pain response. The end goal is to overcome the barriers that prevent functional status improvement, a healthy lifestyle, and reaching their personal goals.

Summary Chronic pain is a major public health issue. Cognitive functional therapy (CFT) and cognitive behavioral therapy (CBT) are both multidimensional psychological approaches to combat the mental portion of difficult pain control. While these therapies are quite different in their approach, they lend to the idea that chronic pain can and should be targeted using coping mechanisms, helping patients understand the pathophysiological process of pain, and altering behavior.
\end{abstract}

This article is part of the Topical Collection on Alternative Treatments for Pain Medicine

Matthew B Novitch

novit018@umn.edu

1 Department of Interventional Pain Medicine, Mt. Sinai, New York City, NY 10016, USA

2 Department of Anesthesiology and Pain Medicine, University of Washington, Seattle, WA 98195, USA 
Keywords Chronic $\cdot$ Pain $\cdot$ Cognitive $\cdot$ Behavioral $\cdot$ Functional $\cdot$ Therapy

\section{Cognitive Behavioral Therapy}

Cognitive behavioral therapy has many uses and has recently been applied to patients with chronic pain in order to alter thought patterns and coping mechanisms as a method of psychotherapy $[1 \bullet, 2]$. Like CFT, CBT focuses on maladaptive cognitions that contribute to emotional distress and functional limitations that result from painful conditions. As a multimodal disorder, pain caused by tissue injury is modulated by the cognitive maladaptation and emotional strain associated with it. Previous studies have shown that CBT is effective for depression, anxiety, stress, and chronic pain [3]. While not only improving chronic pain scores, CBT has been shown to aid functional life, as it has been reported to improve functional status, fibromyalgia, and overall quality of life in addition to changes in self-efficacy, social support, and overall view of life control [4]. There is abundant evidence for the use of CBT in the literature, so much so that multiple meta-analyses have been conducted with frequently positive outcomes.

Chronic low back pain, fibromyalgia, rheumatoid arthritis, and chronic musculoskeletal pain are often the most common disease patterns studied when implementing CBT. Results in large-scale meta-analyses often show small to medium positive effect ranges. Specifically for fibromyalgia, Glombiewski et al. showed that CBT was superior to other psychological treatments for decreasing pain intensity $[1 \bullet]$. Chronic fatigue syndrome has also been shown to benefit in response to CBT, as Malouff et al. revealed a medium effect size in posttreatment fatigue for patients receiving cognitive behavioral therapy against controls [5].

Some groups benefit more from CBT than others, as Pieh et al. [6] described women benefiting more from multimodal pain therapy with a specific focus on CBT-oriented programs compared to their male counterparts. In addition, the Veteran Affairs (VA) developed a project to increase the training and delivery of CBT in their hospitals in 2015. Statistical analysis on the results of this integration showed significant improvements in pain catastrophizing, interference, and quality of life. CBT has also been shown to be effective for those with schizophrenia or those suffering from opiate addiction $[7,8]$. With a promising outlook, more research must be done to develop CBT and identify potential groups that would most improve in order to increase treatment impact.

\section{Cognitive Functional Therapy}

As described above, CFT has a different approach when attempting to alter the pain response. As a multidimensional cognitive framework, an individual is interviewed and key thought patterns and behavioral responses to pain are identified. Four main methods are employed to change the cognitive and functional aspects of an individual's response to pain, namely "making sense of pain," "functional integration," "exposure with control," and "lifestyle change." Each individual pillar of the CFT method has a unique approach to combat pain and improve healthy thought patterns. The data supporting CFT is quite promising and reproducible. It has shown long-term superior efficacy compared to manual therapy, physical therapist-led exercise, and pharmacological interventions. The key outcomes usually identified in studies tend to stray away from the "pain scores" methodology of rating pain, rather focusing on functional and lifestyle improvement $[9,10 \cdot 11,12]$. Qualitative data and case studies support that people benefiting from CFT report that they have a changed mindset toward a more multidimensional understanding of their pain, experience increased pain controllability, and enhanced self-efficacy about achieving functional and lifestyle goals [11].

Making sense of pain often relies on the ability to self-reflect and the willingness of the patient to put their pain into a story, using words and metaphors to consolidate and recreate their journey in a processed form. This process challenges them to reevaluate their previously held beliefs about their pain and the direction they are headed in regards to it. In a non-judgmental, open manner, patients are assisted in identifying contextual factors, unhelpful emotional and behavioral responses to pain, and any cyclical elements that may be causing them to be unable to progress. Exposure with control is an essential component of behavioral change when challenging the pain response. Sympathetic responses to pain, safety behaviors, and functional tasks are targeted during this portion of CFT, which allows individuals to understand the feeling of pain and their physical response to it. As a result, patients gradually adopt levels of conditioning to mechanical stimuli as time goes on, with the goal of ablating fear-avoidance beliefs and behaviors. A reset of the mind when discussing previous experiences that were deemed "painful" or "harmful" and revisiting them after functional conditioning slowly allows patients to become more active and get back to a more active lifestyle. Pain flares are a result of returning to increased activity are an opportunity to reinforce the new ways of thinking and responding to pain. Avoidance of certain tasks or movements due to pain should be addressed immediately as they occur.

These methodologies of CFT eventually lead to a lifestyle change. Exercise programs, normalization of movement, avoidance of sedentary behaviors, and regulating sleep cycles are all implemented as pain is better understood and addressed. The scientific literature largely supports CFT in the treatment of chronic pain, with most trials focusing on chronic low back pain. One recent RCT showed CFT approaches are 
more effective than combining manual therapy and exercise, although the latter was still recommended as a multimodal pain approach. Similar results have been reported in patients with "high-risk" chronic pain, that is, patients with failure of multiple other pain reduction modalities [13].

CFT has also led to greater reductions in disability compared to group exercise and education intervention in those with chronic low back pain [13]. Interestingly, the British Military published a study in 2015 on CFT, as the most common reason for presentation in their armed forces is low back pain. The UK Defense Medical Rehabilitation Centre developed a CFT approach for spinal rehabilitation in conjunction with the NIH. A prospective observational study with 238 participants showed that there were significant improvements in endurance (MSLT), range of motion, kinesiophobia, pain-related lifestyle interference (BPILifestyle), anxiety, and depression $(p \leq 0.001)$ [12].

\section{Future Directions}

With a robust set of data, one can conclude that CBT and CFT are exceptional therapeutic methods in improving chronic pain or the overall well-being of our patients. With most of the therapy being conversational or evaluative in nature and the therapies themselves being tailorable to the individual, the options for expanding and modified CBT and CFT are numerous. One particularly interesting development is the creation of internet-based therapies (iCBT or iCFT). Especially during these times where the 2019 novel coronavirus has decimated the world economy and greatly reduced the ability for many to travel, a remote option for therapy in our chronic pain patients is greatly needed. In addition, the World Health Organization estimated that 34 million people suffering from major depressive disorder go untreated in Europe and the Americas alone [14]. Several studies support the use of iCBT for depression and other psychiatric conditions. These programs and newage methodologies of healthcare and pain relief may help address barriers that hinder access to effective treatment [14].

A growing movement in the world of pain is focused on increasing functionality and mental status rather than focusing on pain scores and numerical measures. Perception of pain has become a major target of our clinical focus which aids in potentially decreasing pharmacologic consumption, invasive procedures, and unhealthy patient habits that may have led to chronic pain [15]. Future research should focus on these mechanisms of controlling chronic pain, as we attempt to decrease our healthcare costs as a country and community. In addition, more conditions should be considered when implementing CBT and CFT, as these treatment modalities consistently show positive effect sizes over several different disease mechanisms. Finally, there is a need for an updated, universally accepted methodology to direct future research as provider to provider variability is an uncontrolled, possibly troublesome variable.

\section{Declarations}

Conflict of Interest The authors declare no competing interests.

Human and Animal Rights and Informed Consent This article does not contain any studies with human or animal subjects performed by any of the authors.

\section{References}

Papers of particular interest, published recently, have been highlighted as:

- Of importance

•- Of major importance

1. Glombiewski JA, Holzapfel S, Riecke J, et al. Exposure and CBT for chronic back pain: An RCT on differential efficacy and optimal length of treatment. J Consult Clin Psychol. 2018;86(6):533-45. https://doi.org/10.1037/ccp0000298 High importance, limited RCTs on this subject and this recent study is vital for the evidence supporting this article.

2. Schemer L, Schroeder A, Ørnbøl E, Glombiewski JA. Exposure and cognitive-behavioral therapy for chronic back pain: an RCT on treatment processes. Eur J Pain. 2019;23(3):526-38. https:// doi.org/10.1002/ejp.1326.

3. Cherkin DC, Sherman KJ, Balderson BH, Cook AJ, Anderson ML, Hawkes RJ, et al. Effect of mindfulness-based stress reduction vs cognitive behavioral therapy or usual care on back pain and functional limitations in adults with chronic low back pain: a randomized clinical trial. JAMA. 2016;315(12):1240-9. https://doi.org/10. 1001/jama.2016.2323.

4. Pincus T, Kent P, Bronfort G, Loisel P, Pransky G, Hartvigsen J. Twenty-five years with the biopsychosocial model of low back pain-is it time to celebrate? A report from the twelfth international forum for primary care research on low back pain. Spine. 2013;38: 2118-23.

5. Malouff JM, Thorsteinsson EB, Rooke SE, Bhullar N, Schutte NS. Efficacy of cognitive behavioral therapy for chronic fatigue syndrome: a meta-analysis. Clin Psychol Rev. 2008;28(5):736-45. https://doi.org/10.1016/j.cpr.2007.10.004.

6. Morley S, Eccleston C, Williams A. Systematic review and metaanalysis of randomized controlled trials of cognitive behaviour therapy and behaviour therapy for chronic pain in adults, excluding headache. Pain. 1999;80(1-2):1-13. https://doi.org/10.1016/ S0304-3959(98)00255-3.

7. Hoffman BM, Papas RK, Chatkoff DK, Kerns RD. Meta-analysis of psychological interventions for chronic low back pain. Heal Psychol. 2007;26(1):1-9. https://doi.org/10.1037/0278-6133.26.1. 1.

8. Pieh C, Altmeppen J, Neumeier S, Loew T, Angerer M, Lahmann C. Gender differences in outcomes of a multimodal pain management program. Pain. 2012;153(1):197-202. https://doi.org/10. 1016/j.pain.2011.10.016.

9. Rathod S, Kingdon D, Weiden P, Turkington D. Cognitivebehavioral therapy for medication-resistant schizophrenia: a review. J Psychiatr Pract. 2008;14(1):22-33. https://doi.org/10. 1097/01.pra.0000308492.93003.db.

10. O'Keeffe M, O'Sullivan P, Purtill H, Bargary N, O'Sullivan K. Cognitive functional therapy compared with a group-based exercise and education intervention for chronic low back pain: a multicentre randomised controlled trial (RCT). Br J Sports Med. 2020;54(13): 
782-9. https://doi.org/10.1136/bjsports-2019-100780 Critical reference defining the need for multimodal therapy, not just exercise and education about pain, but critical mental intervention as well.

11. Vibe Fersum K, Smith A, Kvåle A, Skouen JS, O'Sullivan P. Cognitive functional therapy in patients with non-specific chronic low back pain - a randomized controlled trial 3-year follow-up. Eur J Pain. 2019;23(8):1416-24. https://doi.org/10.1002/ejp.1399.

12. Vibe Fersum K, O'Sullivan P, Skouen JS, Smith A, Kvåle A. Efficacy of classification-based cognitive functional therapy in patients with non-specific chronic low back pain: a randomized controlled trial. Eur J Pain (UK). 2013;17(6):916-28. https://doi.org/ 10.1002/j.1532-2149.2012.00252.x.

13. Conway D, Ladlow P, Ferreira J, Mani-Babu S, Bennett AN. Cognitive functional therapy (CFT)-based rehabilitation improves clinical outcomes in UK military personnel with persistent low back pain. J R Army Med Corps. May 2019;166:336-41. https:// doi.org/10.1136/jramc-2018-001136.

14. O'Keeffe M, Hayes A, McCreesh K, Purtill H, O'Sullivan K. Are group-based and individual physiotherapy exercise programmes equally effective for musculoskeletal conditions? A systematic review and meta-analysis. Br J Sports Med. 2017;51(2):126-32. https://doi.org/10.1136/bjsports-2015-095410.

15. Webb CA, Rosso IM, Rauch SL. Internet-based cognitive-behavioral therapy for depression: current progress and future directions. Harv Rev Psychiatry. 2017;25(3):114-22. https://doi.org/10.1097/ HRP.0000000000000139.

Publisher's Note Springer Nature remains neutral with regard to jurisdictional claims in published maps and institutional affiliations. 